\title{
Rapid assessment and simulation study of the physiological and psychological state of coal miners
}

\author{
JIN Gang ${ }^{* 1}$, ZHANG JinSuo ${ }^{1,2}$, HUANG YiXin ${ }^{2}$ \\ ${ }^{1}$ College of Management, Xi' an University of Science and Technology, Xi'an, Shanxi, 710054, China \\ ${ }^{2}$ School of Economics and Management, Yan'an University, Yan'an, Shanxi, 716000, China
}

\begin{abstract}
In order to achieve a rapid evaluation of the physiological and psychological state of miners and to solve the problem of how to prevent human-caused accidents underground. Based on the existing literature and expert interviews, a rapid evaluation index system consisting of 2 aspects of physiological state and psychological state, 11 indicators such as body temperature, blood pressure and heart rate is established, and the index weights are determined by using AHP hierarchical analysis. Based on the weights, the physiological and psychological status of coal miners is classified into four levels: excellent, good, medium, and poor. Evaluation criteria also is established. A fuzzy comprehensive evaluation model of coal miners' physiological and psychological states is constructed, and the subordination suitable for the evaluation of coal miners' physiological and psychological states is given. Degree function. Using MATLAB2016a to randomly simulate 1000 sets of index data and perform evaluation and analysis, the results found that the proportions of evaluation as excellent and good are $36 \%$ and $35 \%$, respectively; the proportions of neutral and poor are $16 \%$ and $13 \%$, respectively. The evaluation of the physiological and psychological states of five coal miners in a coal mine in Shaanxi Province, with all results above "good", is verified to be consistent with reality. Through simulation analysis and example verification, it is shown that the model is reasonable and applicable to the group of coal miners, and is expected to provide theoretical support for the development of subsequent rapid assessment software systems.
\end{abstract}

\section{Introduction}

Underground accidents in coal mines can cause great harm to coal miners and their families, as well as huge losses to the production and operation of coal mining enterprises. At present, there are still difficulties in the application of unmanned underground mining technology, and a large number of coal miners are still required to carry out operations underground. Investigations into various coal mine accidents have shown that the human factor is the main cause of coal mine accidents in China. The underground environment is complex and still characterized by dust, noise and narrow working surfaces, and underground work requires coal miners to be in a good physiological and psychological state. Therefore, a rapid assessment of the physiological and psychological state of coal miners before going underground can effectively identify potentially dangerous coal miners and reduce the occurrence of underground safety accidents, so it is urgent to use scientific methods to detect and evaluate the physiological and psychological state of coal miners before going underground.

Research on the physiological and psychological state of coal miners has already yielded some results. From the perspective of the physiological and psychological state of coal miners, physiological indicators such as heart rate, blood pressure, blood glucose, respiration rate and electromyography have been concluded to be the main factors affecting the physiological state of coal miners. Work fatigue scales ${ }^{[1-4]}$. Most of the studies have focused on analysis the effects of physiological and psychological indicators on human physiological and psychological states, or on real-time detection and early warning of coal miners' physiological states. As real-time warning is difficult to apply in the underground, the rapid detection and evaluation of the physiological and psychological state of coal miners before they go underground is useful for preventing human errors in the underground. The results of the rapid assessment of the physiological and psychological state of coal miners before going underground are obtained through the evaluation of their psychological state by means of a scale and rapid physiological index data testing before going underground. Based on the results, interventions such as compulsory shift changes and breaks are made for coal miners with poor physiological and psychological status. This method of evaluation enables the prevention of human error in underground mines and reduces the occurrence of man- 
made accidents in underground mines.

\section{Establishment of a rapid evaluation indicator system}

\subsection{Analysis of factors influencing the physiological and psychological state of coal miners}

The physiological parameters of human beings in different states are significantly different, so certain physiological and psychological indicators of coal miners can be tested to evaluate their current physiological and psychological state, and thus to warn of abnormalities and prevent accidents in underground mines. In terms of physiological indicators, heart rate and respiratory rate can significantly reflect a miner's physiological state and are quick and easy to detect. Blood pressure and blood glucose reflect the physiological and psychological state of the miner and are easy to detect, making them important parameters for evaluating the physiological and psychological state of the miner ${ }^{[5]}$. Brain waves are reflected by the body's function, but they can be easily influenced by other factors and the measurement method is cumbersome and does not meet the requirements of rapid testing. Through the above analysis and practical application problems, the following indicators can be summarized to objectively evaluate the physiological state of coal miners, respectively: body temperature, blood pressure, blood oxygen saturation, blood sugar, lung capacity, heart rate, respiratory rate seven indicators. In terms of psychological indicators, the evaluation indicators of coal miners' psychological state mainly include three indicators, namely work stress ${ }^{[6]}$, work fatigue and work burnout. The coal miners' psychological state was evaluated using internationally used and mature scales, namely the Perceptual Stress Scale (CPSS), the Three-Dimensional Fatigue Inventory (3D-WFI) ${ }^{[7]}$ and the Work Burnout Inventory (MBI-GS), to evaluate the three psychological state indicators.

\subsection{Establishment of a rapid evaluation indicator system}

The suitability and competence of coal miners for underground work requires a good physiological and psychological state, which relates to the coal miners' ability to perform their role or specific work activities safely and productively. Based on basic research on the factors influencing coal miners' physiological and psychological states, the factors influencing coal miners' different physiological states were considered comprehensively, and the factors influencing coal miners' physiological and psychological states were summarized through literature analysis and the Delphi method based on literature combing ${ }^{[8-9]}$ and expert interviews. An index system for evaluating the physiological and psychological state of coal miners was established, as shown in Figure 1.

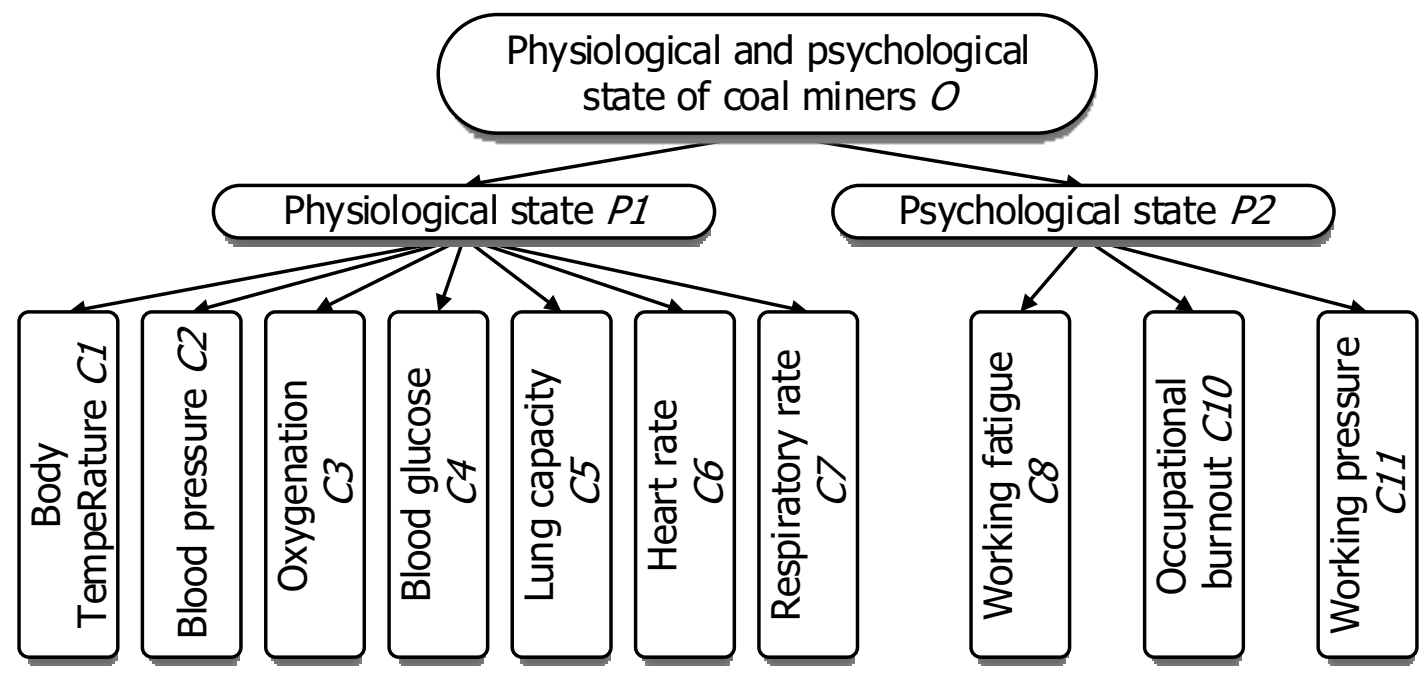

Fig. 1 Evaluation index system of coal miners' physiological and psychological state

\section{Fuzzy integrated evaluation of coal miners' physiological and psychological state}

The physiological and psychological status of coal miners is restricted by many factors and these evaluation factors are fuzzy. Therefore, it is difficult to evaluate the physiological and psychological status of coal miners in a simple quantitative way. In the assessment of physiological parameter index grade, there is a problem that the critical point index value is close to but the assessment grade is far from each other. Fuzzy comprehensive evaluation provides a reasonable and effective way for the scientific evaluation of coal miners 'physiological and psychological state.

\subsection{Establish a set of evaluation indicators}

On the basis of the evaluation index system of coal miners' physiological and psychological state, the evaluation factors of coal miners' physiological and psychological state are divided into four grades and three grades fuzzy 
comprehensive evaluation method is adopted.

The first level evaluation index set: The coal miners' physiological and psychological state $\mathrm{O}$ is divided into two criteria $P_{i}(\mathrm{i}=1,2)$, namely $\mathrm{O}=$ \{physiological state P1, psychological state P2\}.

Second level evaluation index set: each index of $\mathrm{C}$ layer contains 10 indexes $C_{i}(i=1,2 \ldots \ldots 10)$ as index layer.

Third Level Evaluation Index set: Blood pressure $C_{2}=\left\{\mathrm{SBPC}_{21,}, \mathrm{DBP} C_{22}\right\}$.

\subsection{Establishment of the judgment set}

On the basis of determining the grading index set, the evaluation criteria of coal miners' physiological and psychological state are divided into four grades: excellent, good, medium and poor. With reference to the World Health Institute standards and other relevant medical standards, the evaluation standards for the types of coal miners shall be formulated, and the grading of indicators shall be as shown in tables 1 and 2 .

Tab.1 Standards of physiological index grade

\begin{tabular}{|c|c|c|c|c|c|c|c|c|}
\hline \multirow[b]{2}{*}{ Grade } & \multicolumn{2}{|c|}{ Blood pressure } & \multirow{2}{*}{$\begin{array}{c}\text { Blood } \\
\text { glucose } \\
(\mathrm{mmol} / \mathrm{L})\end{array}$} & \multirow[b]{2}{*}{$\begin{array}{l}\text { Heart rate } \\
\text { (cpm) }\end{array}$} & \multirow{2}{*}{$\begin{array}{c}\text { Oxygenatio } \\
\text { n (\%) }\end{array}$} & \multirow{2}{*}{$\begin{array}{l}\text { Respiratory } \\
\text { rate }(\mathrm{cpm})\end{array}$} & \multirow{2}{*}{$\begin{array}{c}\text { Body } \\
\text { Temperatu } \\
\text { re }\left({ }^{\circ} \mathrm{C}\right)\end{array}$} & \multirow{2}{*}{$\begin{array}{l}\text { Lung } \\
\text { capacity }\end{array}$} \\
\hline & $\begin{array}{c}\text { SBP } \\
(\mathrm{mmHg})\end{array}$ & $\mathrm{DBP}(\mathrm{mmHg})$ & & & & & & \\
\hline excellent & $90 \sim 139$ & $60 \sim 89$ & $3.6 \sim 5.6$ & $60 \sim 100$ & $95 \sim 97$ & $12 \sim 20$ & $36.2 \sim 37.2$ & $4500 \sim 5000$ \\
\hline good & $140 \sim 159$ & $90 \sim 99$ & $5.6 \sim 6.1$ & $90 \sim 120$ & $92 \sim 95$ & $<12,20 \sim 25$ & $\begin{array}{c}<36.2,37.4 \\
\sim 38\end{array}$ & $3750 \sim 4500$ \\
\hline medium & $160 \sim 179$ & $100 \sim 109$ & $6.1 \sim 7.0$ & $120 \sim 180$ & $90 \sim 92$ & $25 \sim 30$ & $38.1 \sim 39$ & $3250 \sim 3750$ \\
\hline poor & $\geq 180$ & $\geq 110$ & $\geq 7.0$ & $180 \sim 200$ & $<90$ & 30 & $>39.1$ & $<3250$ \\
\hline
\end{tabular}

Tab.2 Standards of psychological index grade

\begin{tabular}{cccc}
\hline Grade & $\begin{array}{c}\text { Working } \\
\text { fatigue }\end{array}$ & $\begin{array}{c}\text { Occupational } \\
\text { burnout }\end{array}$ & $\begin{array}{c}\text { Working } \\
\text { pressure }\end{array}$ \\
\hline excellent & $1 \sim 2$ & $<50$ & $14 \sim 28$ \\
good & $2 \sim 3$ & $50 \sim 75$ & $28 \sim 42$ \\
medium & $3 \sim 4$ & $75 \sim 100$ & $42 \sim 66$ \\
poor & $4 \sim 5$ & $>100$ & $66 \sim 70$ \\
\hline
\end{tabular}

\subsection{Determination of the weight of evaluation index based on AHP}

For the determination of the weight of factors affecti ng the physiological and psychological state of coal miners, 8 experts and scholars in the fields of medic ine, coal miners' safety research and coal mine safet $\mathrm{y}$ research were selected to score according to the $\mathrm{s}$ cale of 1-9, the authority of the experts was unanim ous. After getting the score of each expert, the judg ement matrix is established and the order of each ex pert is arranged and the consistency test is carried o ut. $\mathrm{CR}=0.0417<0.1$, which satisfies the consisten cy test. In summary, $\mathrm{W}^{\mathrm{T}}=(0.1737,0.1238,0.0692,0.143$ $2,0.0669,0.0723,0.0730,0.1271,0.0416,0.1091)^{\mathrm{T}}$.It can b e seen that body temperature is the most important $f$ actor to affect the physiological and psychological st ate of coal miners, and blood pressure, blood sugar, work fatigue and work stress are also important fact ors.

\subsection{Establishment of membership function}

The creation of membership functions is the key to fuzzy integrated evaluation. After fuzzy processing, the evaluation indexes of the physiological and psychological state of coal miners are divided into four grades: excellent, good, medium and poor. For indicators such as blood pressure, blood sugar, heart rate and body temperature, the ideal value is the median of each grade interval, and the degree of membership of the median of the four grade intervals is set to "1". The membership function for spirometry, the larger the better, should be the opposite of the membership function for blood pressure. On this basis, the membership functions (1)-(4) correspond to excellent, good, moderate and poor respectively.

$$
\begin{gathered}
f(x)=\left\{\begin{array}{l}
\frac{x-a}{\mu_{1}-a}, a \leq x \leq \mu_{1} \\
\frac{\mu_{2}-x}{\mu_{2}-\mu_{1}}, \mu_{1} \leq x \leq \mu_{2} \\
0, x<a \& \mathrm{x} \geq \mu_{2}
\end{array}\right. \\
f(x)=\left\{\begin{array}{l}
\frac{x-\mu_{1}}{\mu_{2}-\mu_{1}}, \mu_{1} \leq x \leq \mu_{2} \\
\frac{\mu_{3}-x}{\mu_{3}-\mu_{2}}, \mu_{2} \leq x \leq \mu_{3} \\
0, x<\mu_{1} \& \mathrm{x} \geq \mu_{3}
\end{array}\right. \\
f(x)=\left\{\begin{array}{l}
\frac{x-\mu_{2}}{\mu_{3}-\mu_{2}}, \mu_{2} \leq x \leq \mu_{3} \\
\frac{\mu_{4}-x}{\mu_{4}-\mu_{3}}, \mu_{3} \leq x \leq \mu_{4} \\
0, x<\mu_{2} \& \mathrm{x} \geq \mu_{4}
\end{array}\right.
\end{gathered}
$$




$$
\begin{gathered}
f(x)=\left\{\begin{array}{l}
\frac{x-\mu_{3}}{\mu_{4}-\mu_{3}}, \mu_{3} \leq x \leq \mu_{4} \\
0, a \leq x<\mu_{3} \\
1, x<a \& \mathrm{x}>\mu_{4}
\end{array} \quad\right. \text { The Fuzzy Comprehensive Evaluation model is as fol } \\
S=W \circ R=\left(w_{1}, w_{2}, \ldots \ldots w_{n}\right)\left(\begin{array}{cccc}
r_{11} & r_{12} & \ldots & r_{1 n} \\
r_{21} & r_{221} & & r_{2 n} \\
& \vdots & \ddots & \vdots \\
r_{n 1} & r_{n 2} & \cdots & r_{n n}
\end{array}\right)=\left(s_{1}, s_{2}, \ldots \ldots s_{1}\right)
\end{gathered}
$$

The Fuzzy Comprehensive Evaluation model is as follows:

Formula, S for the evaluation of the results vector, "o" for the fuzzy operator. W is the evaluation index weight vector, $\mathrm{R}$ is the evaluation index membership matrix. The evaluation result $s_{j}(j=1,2, \ldots \ldots n)$ is obtained by the operation of column $\mathrm{J}$ of $V$ and $R$, which indicate the membership degree of the evaluated object to a fuzzy subset on the whole.

Based on the classification of evaluation indexes, the fuzzy comprehensive evaluation is divided into three levels. According to the formula 5, the fuzzy evaluation results of the first and second levels are calculated respectively, and the final three-level evaluation results of the target level are calculated in the same way. According to the principle of maximum subordinate degree, the evaluation results of coal miners' physiological and psychological state are obtained.

\section{Simulation research on evaluation model of coal miners 'physiological and psychological state}

\subsection{Model simulation}

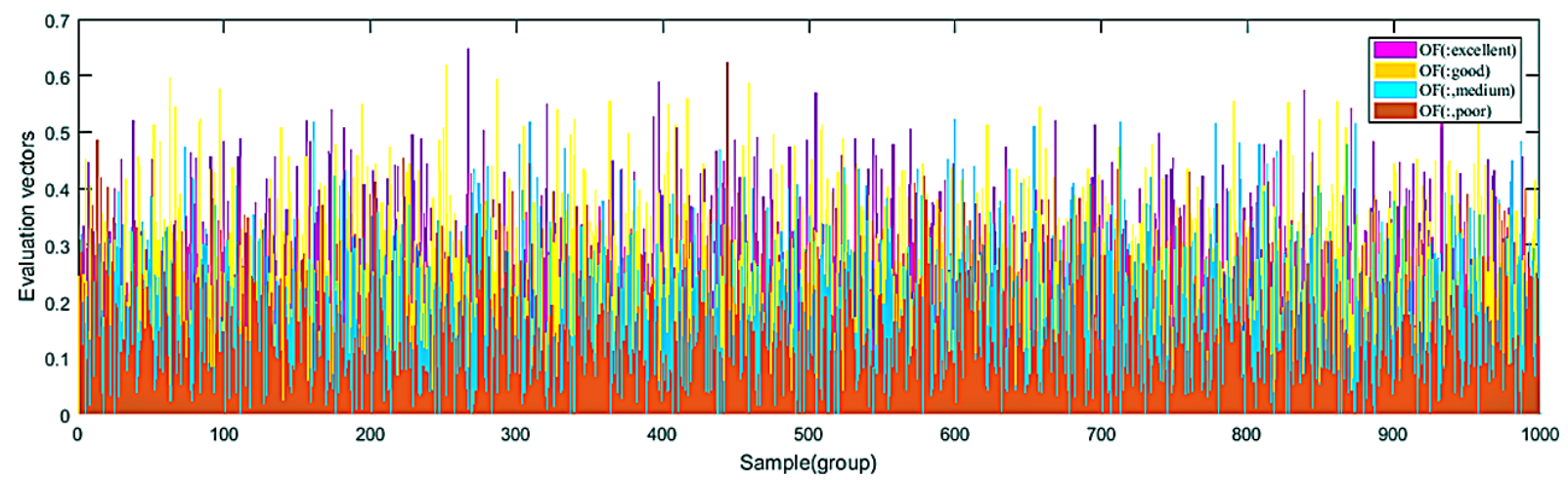

Fig. 2 Image for evaluating vector distribution
The final evaluation result was determined according to the principle of maximum subjection. The proportion of excellent, good, medium and poor evaluation results were $33.6 \%, 35 \%, 21.2 \%$ and $10.2 \%$ respectively.

\subsection{Discussion}

As can be seen, $69.7 \%$ of the sample rated the results as "excellent" and "good", while 31.3\% rated them as "fair" and "poor". ". The simulation results reflect that around $70 \%$ of the miners' physiological and psychological states were within the normal range.
The parameters of physiological indicators and scores of psychological scales of coal miners are randomly distributed in the interval range, so multiple sets of indicator data can be simulated by Monte Carlo method for validation analysis. For the above proposed fuzzy comprehensive evaluation method of coal miners' physiological and psychological status, MATLAB2016a software was used to simulate and analysis the feasibility of the method. The simulation process was carried out in three main steps, firstly, the data matrix R0 was generated for 1000 groups of 12 indicators in the indicator interval range, R0 was a random matrix of 12 columns and 1000 rows generated randomly; secondly, the eigenvectors of 12 columns generated for each group were calculated by the given affiliation function to calculate the affiliation matrix "ro" for each group of indicators, "ro" was the calculated affiliation matrix of 12 rows and 4 columns The final $\mathrm{O}$-vector of the randomly generated matrix was output by multiplying the affiliation matrix with the weight values to the evaluation vector, and the evaluation vector of the physiological and psychological state of the coal miners was obtained through the three-stage comprehensive evaluation. 1000 groups of indicators were distributed as shown in Figure 2.
The evaluation criteria for each indicator are given in conjunction with the medical health assessment and the actual conditions of the miner's type of work. The set of criteria lays the foundation for fuzzy comprehensive evaluation, and the constructed affiliation function can effectively solve the fuzzy problems in the evaluation of indicators. The proposed evaluation model can provide a reasonable solution to the problem of rapid evaluation of the physiological and psychological status of miners, and the evaluation results can be used as an important basis for judging the physiological and psychological status of miners, providing a theoretical basis for the subsequent 
rapid evaluation system of the physiological and psychological status of miners.

\subsection{Interventions}

The article gives the corresponding interventions based on the principles of accident pre-control and the set of evaluation criteria

Compulsory rest and shift change. After evaluating the physiological and psychological state of miners, miners with a "poor" result should be advised to take rest and change shifts. This helps to reduce the risk of accidents by controlling at source the hazards that may occur underground.

Provide safety warnings. After evaluating a miner's health indicators, miners with a "medium" result should be given a safety warning. This is a way to strengthen the miners' own safety awareness and to prevent possible mistakes and dangerous operations from occurring at the source, thus improving the safety management of the mine.

\section{Summarize}

The article constructs a rapid evaluation index system for the physiological and psychological status of miners. By combing through the literature on the physiological and psychological status of miners, a rapid evaluation index system for the physiological and psychological status of miners was constructed, consisting of 10 evaluation indexes: body temperature, blood pressure, oxygen saturation, blood glucose, heart rate, respiratory rate, lung capacity, work stress, work fatigue and work burnout, and the index weights were determined by using hierarchical analysis. The weights were ranked as follows: body temperature $>$ blood sugar $>$ work fatigue $>$ blood pressure $>$ work stress $>$ respiratory rate $>$ heart rate $>$ Oxygenation $>$ lung capacity $>$ work burnout.

The evaluation criteria of the indicators were established and the evaluation model was proposed. On the basis of the constructed index system, the evaluation model of miners' physiological and psychological status based on fuzzy comprehensive evaluation was proposed. Using MATLAB2016a software, 1000 sets of index data within the standard range were randomly simulated for evaluation and analysis, and the results showed that $34.1 \%$ and $35.6 \%$ of the evaluation results were excellent and good respectively; $19.4 \%$ and $10.9 \%$ were medium and poor respectively. Taking five miners in a mine in Shaanxi as an example, the evaluation of the physiological and psychological state of the five miners was conducted and the evaluation results were in line with the reality.

By evaluating the physiological and psychological status of miners, it is possible to screen out miners with poor physiological and psychological status from the source and ensure that all miners in the mine have good physiological and psychological status.

\section{Reference}

1. Svensson, E. A. I., \& Wilson, G. F. (2002). Psychological and psychophysiological models of pilot performance for systems development and mission evaluation. J. 12(1), 95-110.

2. József Varga, Nagy, I., László Szirtes, \& János Pórszász. (2016). Physiological strain in the hungarian mining industry: the impact of physical and psychological factors. J.29(4), 597-611.

3. Yang D. (1997) Analysis of human error causes and control countermeasures. J.(01):17-20.

4. Li Jizuo, Kang Lixun, Zhou Zhili, et al. (2004) Research on psychological assessment of coal mine safety personnel. J.(03):11-14+1.

5. A., K., Ghosh, A., \& Bhattacherjee. (2013). Predictors of occupational injuries among coal miners: causal analysis. J.

6. Carlene M L,Stephen V H,Anthony R. (2008)Global burden of blood-pressure-related disease, 2001.J. 371(9623).

7. Frone, M. R., \& Tidwell, M. C. O.. (2015). The meaning and measurement of work fatigue: development and evaluation of the three-dimensional work fatigue inventory (3d-wfi). J. 20(3), 273-288.

8. Mullen, J.. (2004). Investigating factors that influence individual safety behavior at work. J. 35(3), 275-285.

9. Alice Constantino Joaquim, Maurício Lopes, Luana Stangherlin, ... \& Ingrid D. Schweigert Perry. (2018). Mental health in underground coal miners. J.(6). 